\title{
Diagnostic management of nonalcoholic fatty liver disease: a transformational period in the development of diagnostic and predictive tools - a narrative review
}

\author{
Natalia Rosso $^{1 \wedge}$, Adam M. Stephenson $^{2}$, Pablo J. Giraudi ${ }^{\wedge}{ }^{\wedge}$, Claudio Tiribelli $^{1} \wedge$ \\ ${ }^{1}$ Fondazione Italiana Fegato, ONLUS Area Science Park Basovizza, Trieste, Italy; ${ }^{2}$ Helena Biosciences, Queensway South, Team Valley Trading \\ Estate, Gateshead, UK \\ Contributions: (I) Conception and design: N Rosso; (II) Administrative support: None; (III) Provision of study materials or patients: None; (IV) \\ Collection and assembly of data: None; (V) Data analysis and interpretation: None; (VI) Manuscript writing: All authors; (VII) Final approval of \\ manuscript: All authors. \\ Correspondence to: Natalia Rosso. Fondazione Italiana Fegato - ONLUS, Bldg Q - AREA Science Park Basovizza, SS14 Km 163,5, 34149 Trieste, \\ Italy. Email: natalia.rosso@fegato.it.
}

\begin{abstract}
NAFLD is an emerging healthcare epidemic that is causing predictable adverse consequences for healthcare systems, societies and individuals. Whilst NAFLD is recognized as a multi-system disease with compound pathways that are both benign and pernicious in their unfolding; NASH is generally understood as a deleterious follow-on condition with path-specific tendencies that progress to cirrhosis, HCC and liver transplantation. Recent evidence is beginning to challenge this interpretation demanding more attention to the personalized nature of the disease and its pathogenesis across multiple different cohorts. This means that we need better diagnostic and prognostic tools not only to capture those 'at risk' disease phenotypes; but for better stratification and monitoring of patients according to their treatment strategies. With the advent of pipeline therapies for NASH underway, the medical profession looks to adopt more accurate noninvasive diagnostic tools that can help to delineate and eliminate NASH histology. This review looks at the search for the killer application revealing this particular moment in time as a transformational period; one that is pushing the boundaries of technology to integrate diverse panels of species through sensitive profiling and multi-omics approaches that cast wide, yet powerful diagnostic nets that have the potential to elucidate pathway specific biomarkers that are personalized and predictable.
\end{abstract}

Keywords: Nonalcoholic fatty liver (NAFL); nonalcoholic steatohepatitis (NASH); non-invasive biomarkers; multi-omics; prediction; stratification

Submitted Jun 15, 2020. Accepted for publication Aug 13, 2020.

doi: $10.21037 /$ atm-20-4723

View this article at: http://dx.doi.org/10.21037/atm-20-4723

\section{NAFLD definition \& epidemiology}

Nonalcoholic fatty liver disease (NAFLD) is a multifactorial and progressive disease with two histological phenotypes: nonalcoholic fatty liver (NAFL) characterized by lipid accumulation with no cellular injury (simple steatosis); and nonalcoholic steatohepatitis (NASH) - the progressive form of the disease characterized by cellular injury, inflammatory infiltrates and possible progression to fibrosis and cirrhosis. The knowledge of the natural history of NAFL is currently evolving. Simple steatosis was believed to be a benign,

^ ORCID: Natalia Rosso, 0000-0002-4251-3547; Pablo J. Giraudi, 0000-0003-2852-6648; Claudio Tiribelli, 0000-0001-6596-7595. 
nonprogressive condition that does not increase the overall or liver-related mortality; and NASH-a distinct and progressive disease with a marked increase in liver-related mortality related to cirrhosis and hepatocellular carcinoma (HCC). Emerging data are challenging the long-lasting beliefs that NAFL is completely benign with no clinical sequelae and that NASH is prognostically and clinically relevant. The natural history of NAFLD is not linear: NAFL progresses to NASH in $10-30 \%$ of cases, whereas about $10-15 \%$ of NASH patients progress to cirrhosis (1). The hallmark lesion of NASH is hepatocellular ballooning, which is a marker of hepatocyte injury and a constituent driver of disease progression to fibrogenic remodeling. As a result, liver biopsy is required to differentiate NAFL from NASH limiting data and understanding on both the prevalence and progression of this complex disease.

NAFLD is associated with obesity, hypertension, type 2 diabetes mellitus (T2DM), insulin resistance (IR), hyperlipidemia, or the sum of several of these parameters that is defined as metabolic syndrome (MS). Indeed, due to the tight correlation with systemic metabolic disorders, it has been recently proposed to use the term metabolic associated fatty liver disease (MAFLD) instead of NAFLD $(2,3)$. In obese patients, the incidence of NAFLD was reported to be around $63-99 \%$. The prevalence of NAFLD varies worldwide but has been «estimated» to be around $10 \%$ in children and $25 \%$ in adults. It is now recognized as the most frequent cause of chronic liver disease worldwide (4). Interestingly children affected by NAFLD are typically asymptomatic, with few or no signs of metabolic syndrome. Pediatricians search for other symptoms such as abdominal pain, fatigue, irritability, headaches, confusion, which are not specific to NAFLD. The absence of specific clinical symptoms makes it difficult to obtain early diagnosisleading to late, incidental diagnosis at the mean age of 11-13 years old (5). Likewise, adult NAFLD is commonly silent with no clinical manifestations nor specific symptoms often leading to diagnosis based on exclusion criteria.

The rising prevalence of adult and childhood obesity foreshadows the impending burden of NAFLD. The lack of effectively licensed pharmacotherapy coupled with low patient compliance to lifestyle changes could—on this trajectory-make NAFLD the most significant reason for liver transplantation by 2030 (6). The annual predicted prevalence of NAFLD in the United States (US) and Europe (i.e. Germany, France, Italy, and United Kingdom), divided according to the stages of the disease (7), indicate that 64 million people in the US have NAFLD with results proportionally similar those in Europe i.e., 52 million. The model also predicts the incidence of NAFLD with 10 million new cases forecast in Europe annually-implicating the additional social, economic and healthcare burdens for societies that attempt to deal with the explosion of this disease. In this context, early diagnosis will contribute to the prevention of the disease and its progression to more severe stages, by reducing the cost-infrastructure of downstream treatment strategies and improving the quality of life in society. To date however-and despite the limitations, the gold standard technique for the diagnosis of NAFLD continues to be liver biopsy. In the present review, we will describe the currently available methods, or those under development, for the non-invasive diagnosis of NAFLD.

\section{Current diagnostic tools}

NAFLD is commonly silent with no clinical manifestations nor specific symptoms, thus the diagnosis of the disease is often based on exclusion criteria. Although NAFL or NASH can be strongly suspected in an individual based on imaging and clinical features (such as the presence of metabolic comorbidities and abnormal lab tests), liver biopsy remains the gold standard for the definitive diagnosis of NASH (Figure 1). However, it is an invasive technique with costs, risks, and subject to bias based on sample variability and observer interpretation-with substantial limitations on repeat testing, follow-up and monitoring, not to mention ethical reasons-as the procedure cannot be performed in all suspected subjects.

Patients with NAFL are thought to be at low risk of adverse consequences and progression to cirrhosis/HCC, or other harmful outcomes such as cardiovascular disease and malignancy. In contrast, the presence of NASH increases the risks of liver and possibly non-liver-related outcomes compared to those patients with NAFL alone. The risk of liver-related mortality in NAFLD grows exponentially as the stage of fibrosis increases (8). However, some studies have highlighted the development of HCC in patients with NAFL even in the absence of fibrosis $(9,10)$. The identification of subjects at risk for NAFL and NASH is imprecise-and although there is significant on-going work, accurate and precise non-invasive markers for the diagnosis of NASH are still to be identified (11).

The majority of individuals with NAFLD are asymptomatic or paucisymptomatic (asthenia, abdominal pain at the upper quadrant). The need for generalized screening and surveillance tools is debatable due to the 


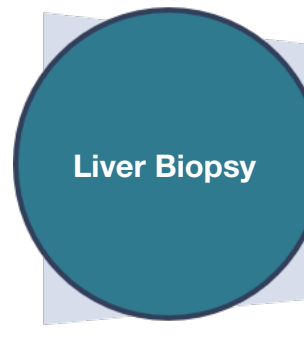

Gold Standard

- Invasive

- Risky

- Costly

- Limitations due to sample's variability observer dependency

Cannot be repeated over the time

Figure 1 Current diagnostic tools. MRI, magnetic resonance imaging; MRE, magnetic resonance elastography; BMI, body mass index.

high direct and indirect costs of diagnostic tests; the low predictive value of surrogate markers (transaminases and non-invasive tests); the risks of liver biopsy and lack of effective treatments. However, it is desirable that the progressive form of NAFLD (NASH), particularly when associated with advanced fibrosis, is correctly identified in patients at risk (age $>50$ years, T2DM, or MS). The anamnesis represents a crucial step in the clinical evaluation of the patient and must be oriented towards the presence of familiarity and comorbidity, stigmata of MS, determination of glucose and lipid profiles. Serology markers of hepatic synthesis (e.g., total bilirubin, albumin, prothrombin time, creatinine), platelet count predictive of portal hypertension, together with increases in ALT (alanine aminotransferase), GGT ( $\gamma$-glutamyltranspeptidase) and an AST (aspartate aminotransferase)/ALT ratio i.e., $<1$ are all biochemical markers that are initially useful in the diagnosis of NASHbut they are all unspecific. Hyperferritinemia is used frequently as a marker of chronic inflammation induced by insulin resistance. In non-diabetic subjects, insulin sensitivity assessment using surrogate indices of insulin resistance derived from blood glucose and insulin levels i.e., Homeostatic Model of Insulin Resistance Index (HOMAIR), could be useful to identify patients at high risk of development of T2DM. Finally, genetic characterization by correlated gene assessment of NASH could be useful in young and lean patients with juvenile NAFLD.

Abdominal ultrasound (US) remains the simplest and most widely used method for detecting hepatic steatosis. Quantification of liver fat could be done with fibroscan CAP modification (Controlled Attenuation Parameter) - a non-invasive measurement tool that is proportional to the attenuation of the ultrasound beam through the liver parenchyma. It can also be used in conjunction with magnetic resonance (MRI) which is now able to quantify limited amounts of intra-hepatocyte triglycerides (TG) and can sample large parenchymal volumes.

However, US has limited sensitivity and does not reliably detect steatosis when it is $<20 \%$ or in individuals with high body mass index (BMI) $\left(>40 \mathrm{~kg} / \mathrm{m}^{2}\right)$. Despite observer dependency, both MRI and US robustly diagnose moderate and severe steatosis when its use is limited by high costs (Figure 1). These techniques allow us to identify and easily quantify intrahepatic TG-the predominant lipids that accumulate in hepatic steatosis. However, TG per se are not hepatotoxic, indeed they have a protective role by providing a buffer against toxic fat, by storage under the neutral form. Besides, it has been proven that there is no difference in the TG content between NAFL and NASH (12). This data seriously reflects on the real utility of TG quantification and the clinically relevant information that can be extrapolated from these findings. Conversely, other reactive lipids have deleterious effects such as lipophosphatidylcholine, ceramides and cholesterol $(12,13)$. However, none of these toxic lipids can be identified nor quantified by any of the previously mentioned techniques.

Another non-invasive technique for the quantification of fibrosis is hepatic transient elastography (TE). The diagnostic accuracy of this technique has been widely validated in patients with $\mathrm{HCV}$ chronic hepatitis, but not in patients with NAFLD. Potential limitations consist of poor sensitivity in mild forms of fibrosis and technical 
difficulty in detecting and interpreting data in the presence of high BMI and/or thoracic fold thickness. Even using an extralarge (XL) probe, the failure rate remains high (35\%). Alternatively, magnetic resonance elastography (MRE) provides a highly accurate measurement of fibrosis, inflammation and steatosis; however, its application in clinical practice is limited by its scarce availability (the instrumentation is only available in academic centers) and its high cost (14) (Figure 1).

In general, the goal is to capture those NASH patients that present early in the NAFL patient pathway. Measurement of the severity of necro-inflammation (grading) and fibrosis (staging) has been standardized and validated in two semiquantitative scoring systems for the histopathological classification of NAFLD i.e., NASH Clinical Research Network (NASH CRN) score and the most recent Steatosis, Activity and Fibrosis (SAF) score. NASH CRN is a valuable research tool but it should not be used to differentiate NAFL from NASH-as underlined by the authors of the original study (15). Moreover, the application of NAS as a prognostic tool in daily practice is controversial $(16,17)$. In contrast, the SAF score uses a simple algorithm to evaluate steatosis, necroinflammatory activity and fibrosis separately - to classify patients as normal, NAFL or NASH (18). The degree of liver fibrosis is the parameter that is optimally associated with general and liver-related mortality $(8,19)$. However, the histological differentiation between NAFL and NASH does not predict fibrosis progression and carries little prognostic information.

To date, the demonstration of histological improvement is a fundamental requirement for the approval of any pharmacological treatment required by the European Medicines Agency (EMA) and the Food and Drug Administration (FDA). In particular, since the FDA does not recognize an indication of treatment in simple steatosis (NAFL), it is believed that therapy should have a histological effect in terms of NASH improvement. The reason for this approach is currently based on data demonstrating a risk of end-stage progression of liver disease that exists only in patients with NASH. Although histology represents the only reliable diagnostic method, high associative costs and non-negligible patient risks limit its use on a large scale. Due to all these limitations, there is still an unmet clinical need to distinguish those individuals with an early disease from those at the highest risk of clinical complications. Thus, to date, new alternatives are urgently needed.

\section{Novel noninvasive diagnostic alternatives}

Among the available serum biomarkers for the assessment of steatosis there are several validated scores such as fatty liver index (FLI) (20), SteatoTest (21) and NAFLD liver fat scores (22) (Figure 2). These scores are associated with insulin resistance (IR) and reliably predict the presence, not the severity, of steatosis. However, in clinical practice, quantification of fat content is not of interest, except as a surrogate of treatment efficacy and is therefore not generally recommended. The diagnosis of NASH instead provides important prognostic information and indicates an increased risk of fibrosis progression, cirrhosis and possibly hepatic comorbidities such as HCC. Nowadays clinical, biochemical or imaging measures cannot distinguish NASH from steatosis.

Regarding fibrosis, many serum markers have demonstrated acceptable diagnostic accuracy, as defined by an area under the receiver operating characteristic curve (AUROC). For example, NAFLD fibrosis score (NFS) (23), Fibrosis 4 calculator (FIB-4) (24), Enhanced Liver Fibrosis (ELF) (25) and Fibrotest (26) predict overall mortality, cardiovascular mortality and liver-related mortality. The tests perform best at distinguishing advanced $>\mathrm{F} 3$ vs. nonadvanced fibrosis but no significant $>\mathrm{F} 2$ or any $>\mathrm{F} 1 v s$. no fibrosis (Figure 2). Considering all these parameters, fibrosis stage is the parameter that best associates with overallrelated and liver-related mortality. Thus, efforts must be devoted especially to the diagnosis of this specific event.

\section{Plasma protein, miRNAs, metabolites}

The analysis of plasmatic metabolites to perform a differential diagnosis of NAFL and NASH is an interesting approach. Studies have reported a relationship between serum uric acid (UA) levels and NAFLD development. In a large study of 100,275 subjects from America and Asia, Darmawan et al. (27) found that the risk of NAFLD was increased almost 2-fold in the group with the highest serum UA compared to the group with the lowest UA (28-30). These findings make UA level (or other metabolites from purine catabolism) an attractive parameter to be included in the various existing non-invasive algorithms to improve specificity in NAFLD diagnosis or for a better disease stratification. In this context, we recently published a study utilizing surface-enhanced Raman spectroscopy (SERS) of plasma (31) demonstrating how the UA/hypoxanthine ratio was significantly different between NAFL and NASH in 
NONINVASIVE ALTERNATIVES

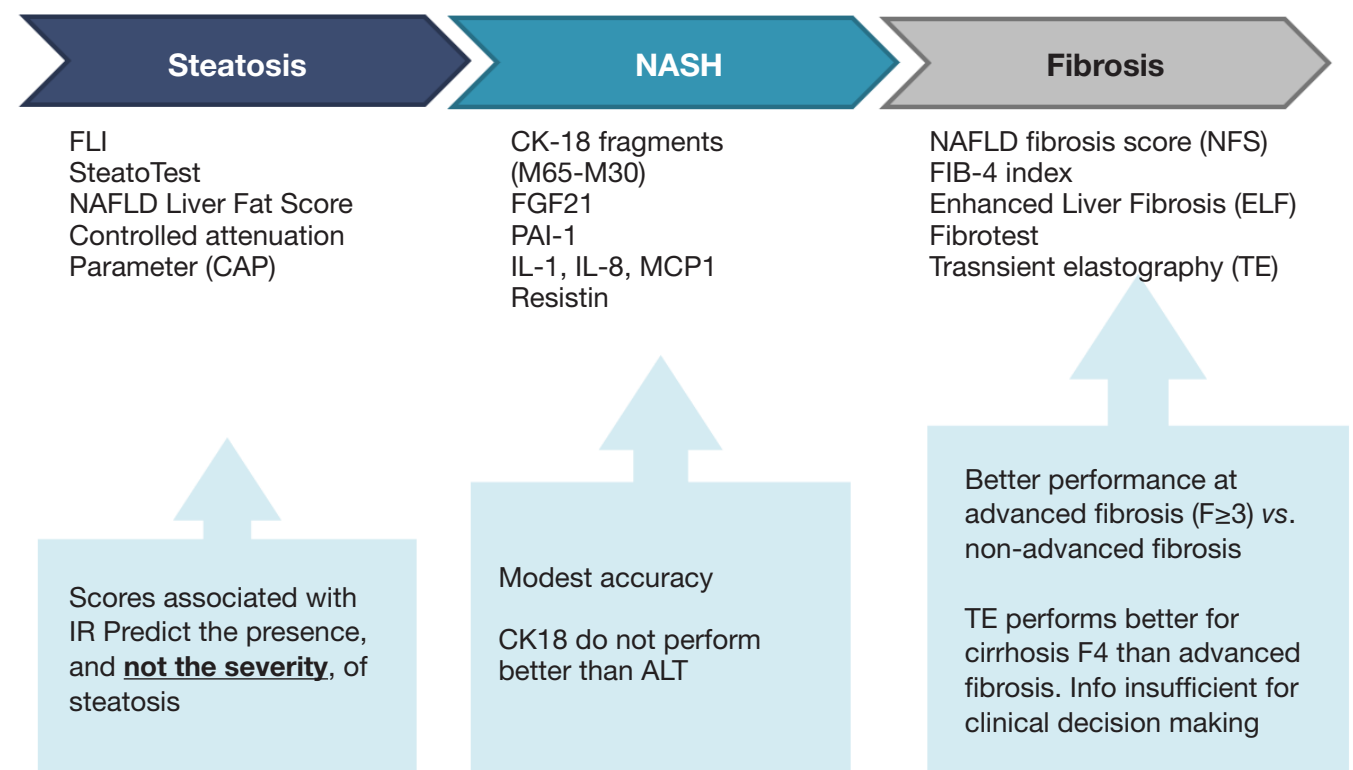

Figure 2 Limitations of the currently noninvasive diagnostic tools. FLI, fatty liver index; IR, insulin resistance; CK-18, cytokeratin 18; FGF21, fibroblast growth factor 21; PAI-1, plasminogen activator inhibitor-1; IL-1, interleukin 1; IL-8, interleukin 8; MCP-1, monocyte chemoattractant protein-1; FIB-4, the fibrosis-4 index.

women (Table 1).

Alternative diagnostic algorithms using different proteins in plasma/serum have been proposed for differential staging of NAFL. Concerning NASH diagnosis, Feldstein et al. (32) reported for the first time that cytokeratin 18 (CK-18) fragment was able to predict NASH in NAFLD patients with an AUC of 0.83 , sensitivity of $75 \%$ and specificity of $81 \%$ (33). Subsequently, CK-18 has been combined with other candidates [such as fibroblast growth factor 21 (FGF21) (34), adipocytokines (35), resistin (36), Fas cell surface death receptor (Fas) (37)], in several panels to improve the robustness of NASH diagnostic testing (Table 1). For a full revision of the diagnostic capabilities of CK-18, FGF21 and their combinations in diagnostic panels have been systematically reviewed by He et al. (45) illustrating the promise of these biomarker combinations.

More recently, promising lists of proteins have been identified using proteomic technologies for biomarker discovery. Among them, we should mention the systematic review performed by Lădaru et al. (46) summarizing 22 studies on NAFLD, but highlighting only 3 serum studies utilizing protein candidates proving NASH efficacy $(38,39,47)$. In consideration of NAFLD fibrosis, procollagen III amino terminal propeptide; (PIIINP), pro- peptide of type III collagen (Pro-C3) and tissue inhibitor of metalloproteinases 1 (TIMP-1) have been widely studied as markers of extracellular matrix turnover and validated as either single or combined panel markers $(40,48)$. Recently, Boyle $e t$ al. reported the development and validation of 2 new panels (FIBC3 and ABC3D) for advanced fibrosis ( $F$ $\geq 3$ ) diagnosis in NAFLD; obtaining in the validation setup diagnostic accuracies of 0.83 and 0.81 respectively (41). As for the utility of circulating miRNAs as markers of fibrosis, Lopez-Riera et al. performed a comprehensive validation study of 18 candidates' miRNAs, previously postulated as biomarkers of NAFLD. The study confirmed three in particular (i.e., miR-27b, miR-16, and mir-30c) that were consistently altered in patients with NASH and severe fibrosis, with miR-30c presenting the most promise with an AUROC value of 0.72 to diagnose severe fibrosis (43). The various biomarkers mainly used for diagnosis of NASH and liver fibrosis in NAFLD are listed in Table 1.

Recently, Hou et al. (49) conducted a plasma proteome screening in patients with early and advanced NASH. Five proteins (i.e., Complement component C7; $\alpha$-2macroglobulin; Complement component C8 $\gamma$ chain; Fibulin-1; $\alpha$-1-antichymotrypsin) were identified as putative biomarkers to differentiate NASH groups, 
Table 1 Novel serum/plasma proposed biomarkers

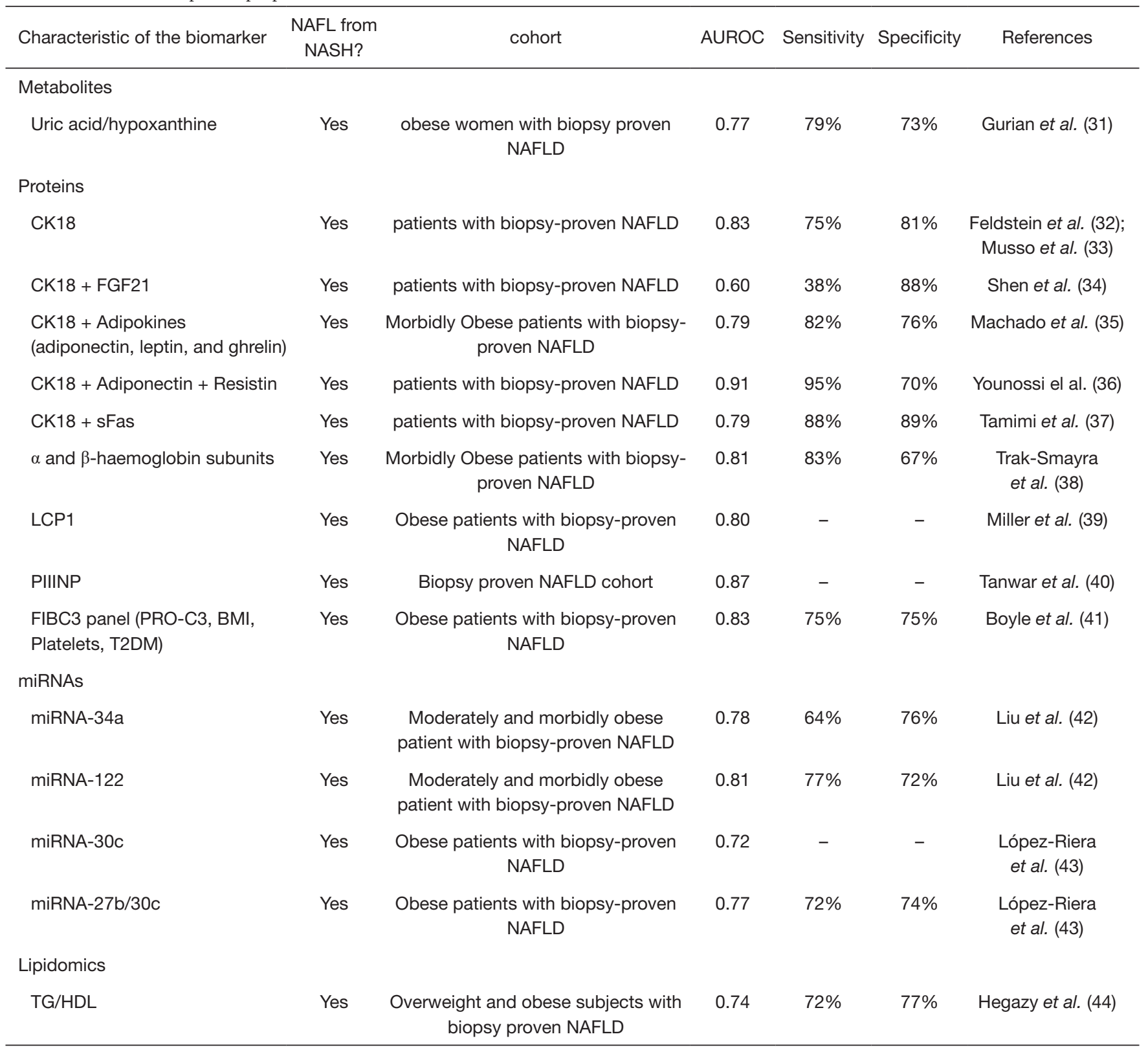

CK-18, cytokeratin 18; FGF21, fibroblast growth factor 21; sFAS, soluble Fas cell surface death receptor; LCP1, lymphocyte cytosolic protein1; PIIINP, procollagen III amino terminal propeptide; ProC3-pro-peptide of type III collagen; BMI, body mass index; T2DM, type 2 diabetes mellitus; TG, triglycerides; HDL, high density lipoprotein-cholesterol.

however-further validation would be required using larger serum cohorts. Additionally, Lai et al. recently evaluated SOMAscan-a novel aptamer-based proteomics platform for diagnosis and monitoring of NASH. The preliminary study evidenced a panel of 7 proteins (C7, CL-K1, IGFBP7, Spondin 1, IL-5Ra, MMP-7, and TSP2) able to discriminate accurately between early and advanced fibrosis in NASH (50).

Concerning circulating miRNAs as biomarkers of $\mathrm{NASH}$, the most recent findings have been examined in two recent systematic and meta-analysis reviews $(42,51)$. The study of Liu et al. included the analysis of thirty-seven miRNA expression profiles and six diagnostic accuracy reports demonstrating the diagnostic capacity of miRNA 
34a, miRNA-122 and miRNA-192 to distinguish NAFL from NASH (42). In the second study, Cai et al. included 23 reports enrolling 1,408 NAFLD and 926 healthy subjects, reporting 7 upregulated miRNAs (i.e., miR-122, miR-99a5p, miR-34a, miR-1290, miR-27b-3p, miR-192-5p and miR-148a-3p) and 5 downregulated miRNAs (i.e., miR197, miR-146b, miR-181d, miR-99a, and miR-29a) with potential applicability as diagnostic markers of NAFLD. Among them, miR-122 showed the highest diagnostic accuracy, but unfortunately, it could not distinguish between NASH and NAFL subjects.

\section{A more predictable disease? Lipidomics, metabolomics and lipotoxicity}

Due to the low prognostic importance attached to intrahepatic TG (IHTG) measurements and the asymmetry between steatosis and necroinflammation at intermediate NAFL scores across different patient cohorts-high baseline IHTG measurements have been strongly suggested for the reporting of NASH resolution in therapy studies (52). Moving away from triglycerides as proxies of DNL (53) and intrahepatic inflammation (52), lipidomics allows a more specific approach in theory by considering those toxic lipid species that emerge at any point in the NAFLD journey. In this sense, lipidomics provides an efficient way of identifying the quantity, role and function of key lipids in the pathogenesis of NAFLD—and in particular, those lipid intermediates that are associated with particular inflammatory cascades. Therefore, various spin-off approaches may allow diagnosing NASH with high accuracy and precision.

Lipidomics is advancing technology towards routine biomarkers capable of predicting and stratifying NASH. However, we see that the main obstacle to clinical adoption is the apparent trade-off between the need for assay sensitive techniques able to detect lipid species at low molecular concentrations; and readily available, userfriendly and inexpensive equipment. Utilizing simple inhouse biomarkers, Hegazy et al. (44) determined the value of cardiovascular risk ratios of TG/HDL, LDL/HDL, total cholesterol/HDL as predictors of $\mathrm{NASH}$ - and the correlation of such ratios with disease severity in overweight and obese subjects with biopsy-proven NAFLD. The authors found that TG/HDL ratio was significantly higher in NASH than in controls. The TG/HDL ratio positively correlated with steatosis, ballooning, inflammation, Body Mass Index (BMI) and NAS (AUROC of $0.74,71.8 \%$ sensitivity, and $76.8 \%$ specificity) (Table 1 ). On the other hand, the use of more sophisticated lipidomic technology i.e., liquid chromatography-mass spectrometry (LC-MS) was employed to measure the serum of 467 patients with biopsy-proven normal liver, NAFL and NASH using a panel of 28 saturated and unsaturated TGs to predict NAFLD from normal liver, and NASH from NAFL (54). As expected, the NAFLD diagnosis was very sensitive with an AUROC of 0.90. On the other hand, NASH data revealed accuracy (0.77), sensitivity (70\%), specificity (81\%) with borderline NASH cases defined and omitted from the data. Whilst both techniques pick-up on the accumulation of TGs, impairment of lipoprotein synthesis and high very low density lipoprotein (VLDL)-cholesterol secretion, which are common features of de novo lipogenesis (DNL) and dyslipidemia, wider questions remain as to whether these available solutions are sensitive enough to replace competing scanning modalities-irrespective of their low or competitive cost.

On the other hand, innovative approaches such as random typing and machine learning of quantified lipid species have attempted to catch all the available species in the NAFLD matrix -in order to obtain the most revealing NAFL and NASH signatures-underpinned by their regulating genes, metabolites and hormones (55-61). Mapping out the ontological heterogeneity of NASH, 32 lipid species were identified consisting mainly of metabolites involved in fatty acid synthesis, oxidation and glycerophospholipid metabolism (60). In a separate study using an MS/machinelearning approach of 32 lipid species, the study revealed the toxic fingerprint of one NASH panel with $100 \%$ sensitivity and specificity that included miristic acid (C14:0), palmitic acid (C16:0), palmitoleic acid (C16:1n-7), vaccenic acid (C18:1n-7) and oleic acid (C18:1n-9) combined (55). Whilst this NASH mix of toxic markers significantly enhanced cell death at low concentrations, this study derived the lipid content from livers and not from blood serum or plasma leaving open the challenge of translating hepatic NASH biomarkers (55). Of 184 hepatic lipid species and 132 plasma lipid species, only 48 of significance are common to both with plasma susceptible to the interfering affects of peripheral extra-hepatic tendencies (58). On the other hand, the 5 toxic lipids found in hepatocytes (55) produced a very similar 5 toxic profile to that found in a recent serum study (58) with a significant NASH profile-with only two of five shared lipids i.e., arachidonic acid and gamma-linolenic acid presenting more significance than either miristic and vaccenic acid (58). 
Whilst more streamlined serum based lipotoxic panels need to be validated, Perakakis et al. demonstrated the accuracy of a 29 lipid panel to predict healthy status, NAFL and NASH (59). The accuracy of these lipids for the classification of healthy $v s$. NAFL $v s$. NASH was highly predictive $0.99,0.94$ and 0.96 respectively-with a sensitivity of $92 \%$ and a specificity of $93 \%$ (59). Specifically, palmitic acid was higher in NAFL and NASH compared to the healthy population. Moreover, the monounsaturated fatty acids cis-palmitoleic acid and oleic acid were higher in NASH compared to the healthy control; whereas linoleic fatty acid and arachidonic fatty acid were lowerrepresenting the possible 'adaptive response' to FA overload (62). This ties in with other work that highlights the changing fatty acid composition of membrane lipids from polyunsaturated fatty acids (PUFAs) through to monounsaturated fatty acids (MUFAs) and saturated fatty acids (SFAs). As the fatty acid (FA) composition of diets monotonously hits the liver there is a change in the lipid membrane content and cardiolipin profile (57) effecting several regulatory pathways including cell signalling and insulin sensitivity-leading to spikes in inflammatory and oxidative stress markers i.e., prostaglandin E2 (PGE2) and 8-iso-prostaglandin F2alpha (PGF2 $\alpha$ ) respectively (56) linked to mitochondrial impairment and beta-cell dysfunction (57).

Oxidative stress is one of the driving forces involved in the progression of NAFLD, thus lipid peroxidation products represent appealing candidates as diagnostic markers, especially to follow NASH progression. Lipid peroxidation products are synthesized via free radicalmediated direct oxidation and enzymatic pathways with lipoxygenases, cyclooxygenases and cytochrome P450. Arachidonic acid (AA), linoleic acid (LA), eicosapentaenoic acid (EPA), or docosahexaenoic acid (DHA) can produce multiple isomers of oxidized fatty acids (eicosanoids), all of which may activate the immune system. The assessment of oxidized fatty acids is an emerging concept for the diagnosis of NASH. Loomba and colleagues (63) carried out a cross-sectional analysis in subjects with NAFL, $\mathrm{NASH}$ and non-NAFLD and they showed that plasma eicosanoid and other PUFA metabolite profiling can differentiate between NAFL and NASH. Specifically, 11,12-dihydroxy-eicosatrienoic acid (11,12-diHETrE) and a panel including 13,14-dihydro-15-ketoprostaglandin $\mathrm{D}_{2}$ (dhk PGD2) and 20-carboxy arachidonic acid (20-COOH AA) demonstrated an AUROC of 1.

Whilst eicosanoid metabolites are emerging as candidate biomarkers of NASH, other studies curiously revealed greater predictive power from plasma sphingolipid species-notably longer chain ceramides e.g., 1-deoxyceramide species i.e., C24:1DH 1-deoxyCer and C26DH 1-deoxyCer (58). Altogether this evidence suggests that toxic lipids could move us away from surrogate biomarkers of NAFLD progression towards specific quantitative panels of lipotoxic species that not only confirm NASH histology with high accuracy, but which also flag up downstream inflammatory pathways linked to metabolite dysregulation, hepatic insulin resistance, mitochondrial dysfunction and oxidative stress regulated by a network of intrahepatic and extrahepatic pathways (64).

\section{Liver glycomics, NAFLD and NASH}

Liver glycomics has recently emerged as a powerful toolbox utilising sophisticated technologies and diagnostic biomarkers for a plethora of disease applications. $\mathrm{N}$-glycans are synthesised in the liver and plasma-B cells-and play significant roles in the structure, function, regulation, signalling, mediation and binding of cellular and protein interactions. We know therefore that glycosylationand aberrant forms thereof-including the formation and abundance of sugars, are closely bound with the pathology of the liver and the topology of disease aetiologies and pathways as they evolve over time (65). In recent history, liver glycomics has emerged to provide novel analytical tools and clinical applications for the typing and quantitation of $\mathrm{N}$-glycans for the prediction of NAFLD (66), NASH (67), fibrosis (68), Child Pugh class A cirrhosis (69), HCC $(70,71)$ and HCC risk (72). These glycan biomarkers have emerged either in the form of a connected profile, or they have originated due to the targeting of specific glycosylated proteins. In this sense, a number of glycan biomarkers have borne fruit in the search for NASH diagnostics e.g., core- and multi-fucosylated glycans (59), agalacto-IgG component (67), fucosylated haptoglobin (66) and mono-fucosylated glycan of alpha-1 antitrypsin: AATA3F (73).

Fucosylated haptoglobin (Fuc-Hpt) has been recognised as a parameter of NASH due to the cellular effects of hepatocyte ballooning, damage to the 'fucosylationmachinery' and elevated secretion of this bile end-product into sera (66). As a hepatocyte ballooning marker, Fuc-Hpt proved to be slightly superior for NAFLD vis a vis NASH albeit with closely aligned performance data for AUROC ( 0.75 vs. 0.73$)$ sensitivity ( $71 \%$ vs. $69 \%)$, specificity $(75 \%$ vs. 
$73 \%)$ and accuracy (72\% vs. 69\%). Fuc-Hpt demonstrated general superiority in the NAFLD cohort in comparison to M30 antigen; however, comparatively more sensitivity $(69 \%$ vs. $42 \%)$ than specificity $(73 \%$ vs. $82 \%)$ in the NASH cohort (66). Other approaches have used a novel immunoprecipitation glycomics method utilising MALDITOF MS to measure the diagnostic utility of tri-antennary tri-sialyated mono-fucosylated glycan of alpha- 1 antitrypsin AAT-A3F (73). This particular glycan is useful because the fucosylation branch emerges at the point of intrahepatic inflammation caused by the upregulation of FUT6 and mediated by pro-inflammatory interleukin-6 (73).

Normally, we expect to find low sensitivity in NASH biomarkers generally, but also narrow cut-points between early and advanced NASH. On the contrary, AAT-A3F demonstrated the potential to diagnose early NASH defined by Brunt stages $0-1$ with high accuracy based on a distinguishable cut-off range between early and advanced stages i.e., 7.9-14.1 $\mu \mathrm{M}$ (73); especially compared to FucHpt where the cut-points for NASH and ballooning were the same (i.e., $36.1 \mathrm{U} / \mathrm{mL}$ ) and closely related, comparatively speaking, to fibrosis severity prediction at $38.8 \mathrm{U} / \mathrm{mL}$ (66). The respective performance of AATA3F comparing early against progressive NASH produced promising AUROC (0.69 vs. 0.68$)$ sensitivity (79\% vs. $38 \%$ ), specificity ( $58 \%$ vs. $95 \%$ ), PPV (56\% vs. $90 \%$ ), NPV (80\% vs. 54\%) data suggesting that AAT-A3F could be used to stratify and monitor 'at risk' NASH patients due it being highly sensitive to lobular inflammation and highly specific at more progressive stages.

Targeting protein glycosylation patterns can reveal pathway specific tendencies, however, a number of profiling techniques are significant $(59,65)$. Using highly sensitive gas chromatography mass spectrometry (GC-MS), 17 glycans of statistical significance were sequenced among a profile of $61-$ with multi and core-fucoslyated glycans containing sialic acids increasing diagnostic accuracyin addition to agalactosylated non-fucoslyated glycans in some instances (59). When we look at the ROC curves for the classification of healthy, NAFL and NASH controls and compare them to those in the obese cohort, a panel of 5 glycans demonstrated AUCs for NAFL (0.72 vs. 0.70) and NASH (0.69 vs. 0.76 ) with greater levels of specificity $(78 \%$ vs. $81 \%$ ) than sensitivity (54\% vs. $64 \%$ ), suggesting higher diagnostic accuracy for NAFLD with greater 'ruling in' potential for NASH - especially in obese cohorts (59).

Curiously, these performance characteristics dramatically improved when integrating lipidomic and glycomic results especially. For example, one 20 lipid-glycan panel provided high sensitivity (89\%) and high specificity (91\%) with a NASH AUC of 0.96; and an even greater sensitivity (91\%) and specificity (95\%) in the obese cohort with an AUC of 0.96. This approach demonstrated the huge potential of glycomics to significantly enhance diagnostic accuracy; but it also revealed the power of a 'multi-omics' approach. By integrating lipid species, $\mathrm{N}$-glycans and metabolites with say hormones and genes-and applying machine learning algorithms to complex data sets, the integrated pathways of NAFLD phenotypes may well become more predictable and prognostic.

In the short term however, we need to be more realistic. The quantification of glycans and other analytes through mass spectrometry methods, unfortunately today-is operationally complex, expensive and not widely available. Notably, it costs $\$ 605$ for one multi-omics test (59) which would far exceed the expectations of clinical laboratoriespublic or private. On the other hand, it is now possible to apply cost-effective glycomics for liver disease diagnosis on affordable capillary electrophoresis systems used routinely in clinical laboratories for high-throughput screening of rare cancers and blood diseases. For example, the Glyco Liver Profile-highlighted elsewhere (65) provides a simple method to cleave, label and then separate $\mathrm{N}$-glycans from serum by automated capillary electrophoresis-providing an all-in-one panel of glycan indices that report separate results for inflammation, fibrosis staging, compensated cirrhosis and HCC risk.

A number of publications have reported on the versatility of this multi-aetiology application-from NAFLD through to paediatric NASH, HCV through to NASH fibrosis, Child Pugh A cirrhosis and HCC (67-69,72,74). Given the range of this multi-parameter toolbox it could be very useful in the context of NAFL patients skipping straight to HCC e.g., $34.8 \%$ in one regional UK case (75)providing an urgent clinical need for sensitive NAFLD tools that can stage fibrosis and predict HCC. In principle, this approach measures an increase of core fucosylated agalactosylated biantennary glycan (e.g., NGA2F) released from IgG in relation to a decrease of galactosylated nonfucoslyated biantennary glycan (e.g., NA2) cleaved from liver synthesised glycoproteins. In previous studies, this approach proved very promising and highly sensitive in relation to NAFLD and NASH with AUCs of 0.74 (76), 0.66-0.75 (77) and 0.72 (67)—where the degree of NASH-related fibrosis was independent of steatosis severity and lobular inflammation (76). Whilst glycomics 
has proved very promising and highly sensitive in relation to NAFLD (59), the use of agalactosylated IgG presents challenges in the context of other inflammatory diseases and interfering co-morbidities (65). On the other hand, it could have a significant role to play in the stratification and monitoring of NAFL patients with significant fibrosis prognosed as either low or high risk of HCC (65).

\section{Conclusions and future directions}

NAFLD is a multi-system disease following a slowprogressive clinical course underpinned by alternating networks of genes, proteins, metabolites, glycans, and hormones, which are also affected by personalized health conditions, dietary habits and environmental factors coinciding to produce pathological ramifications that are, in this disease specifically, inconspicuous and hidden from view. Due to the dynamic characteristics of NAFLD progression, the currently available diagnostic methods are inefficient in depicting the real ongoing situation in the liver, mainly because they all provide a "static picture" of a particular moment in time. By no means is this useful in predicting the progression/regression of NAFL/NASH. Thus, the real challenge for the future is to develop techniques that are able to provide a "dynamic movie" for understanding the ongoing disease pathogenesis.

In the search for non-invasive discriminators of NAFLD and NASH with optimum histological accuracy, no single biomarker is emerging as the 'killer application'. On the other hand, biomarker panels are emerging through the translation pipeline in commercial panels of proteomic, lipidomic and glycomic applications that offer high NAFL sensitivity and NASH specificity for risk-stratification irrespective of fibrosis grading. The development of a methacetin breath test with potential for predicting NASH liver cirrhosis is also an interesting point-of-care (POC) innovation that could be considered for screening (78). Nevertheless, the clinical utility and cost-effectiveness of these new tests will need to be proven against available biomarkers and scanning modalities, though it is encouraging that these specialist tests are becoming available on routine chemistry and POC platforms.

What is perhaps more exciting is the emergence of 'nextgeneration' tools that pin-point lipid species and metabolites involved in down-stream lipogenic and glycolytic pathways that lead to alterations in hepatic insulin sensitivity, inflammatory homeostasis and lipid toxicity (79)—further enhanced by the horizontal integration of cross-correlated multi-omics species involving glycans, genes, hormones, immune-proteins etc., that may afford greater depth and breadth in diagnostic accuracy in ways that are pathway specific. Whilst these developments are exciting, clinical adoption of these products will be challenged by new chemical and in vitro diagnostic (IVD) regulations-in addition to high material costs and technology access.

At the research level, what needs to happen of course is the standardization of techniques, cohorts, diets, analytesso that we may view the picture of NAFLD disease not as a kaleidoscope of fractured patient cohorts and results, but as a movie or library of movies_ of what is a 'multi-hit' disease in motion with different starting and end points so that we may read and predict the script of NAFLD as a multisystem disease with phenotype characteristics that we can diagnose and forecast.

In conclusion, there is a need to rethink the approach by accepting the use of a more efficient, complex and heterogeneous strategy by integrating all the available resources. To tackle this ambitious issue, we need to take advantage of all the available data, thus we need "connectivity" and "blendomics" to obtain information as accurate and complete as possible. Patients would need to be stratified better according to their metabolic, genomic, proteomic, lipidomic, microbiotic changes to create a clearer and more comprehensive picture. If we accept this challenge, perhaps healthcare systems could be mobilized to connect IVD, big pharma, laboratory infrastructures and patients through primary care-including access to untapped longitudinal patient data sets-so that technology translation and therapy development moved as one in an accountable framework with the broader goal of creating predictable disease pathways and personalized treatment strategies.

\section{Acknowledgments}

Founding: PJG is supported by Post-doctoral Fellowships 2020 from Fondazione Umberto Veronesi, Italy. NR is funded by Fondazione Cassa di Risparmio di Trieste (CRTrieste) ProFeGra Project and by Fondazione Italiana Fegato - ONLUS.

\section{Footnote}

Provenance and Peer Review: This article was commissioned by the Guest Editors (Ralf Weiskirchen and Wolfgang Stremmel) for the series "Unresolved Basic Issues in 
Hepatology" published in Annals of Translational Medicine. The article was sent for external peer review organized by the Guest Editors and the editorial office.

Peer Review File: Available at http://dx.doi.org/10.21037/ atm-20-4723

Conflicts of Interest: All authors have completed the ICMJE uniform disclosure form (available at http://dx.doi. org/10.21037/atm-20-4723). The series "Unresolved Basic Issues in Hepatology" was commissioned by the editorial office without any funding or sponsorship. Dr. Rosso reports grants from Fondazione Cassa di Risparmio di Trieste (CRTrieste) ProFeGra Project, during the conduct of the study. Dr. AMS is a Director of Helena Biosciences - an IVD company that manufacturers and supplies the Glyco Liver Profile. The Glyco Liver Profile incorporates the GlycoFibroTest and the GlycoCirrhoTest, which have two issued patents. Dr. PSG reports grants from Fondazione Cassa di Risparmio di Trieste (CRTrieste) ProFeGra Project, personal fees from Post-doctoral Fellowships 2020 from Fondazione Umberto Veronesi, Italy, during the conduct of the study. Dr. CT reports grants from Fondazione Cassa di Risparmio di Trieste (CRTrieste) ProFeGra Project, during the conduct of the study. The authors have no other conflicts of interest to declare.

Ethical Statement: The authors are accountable for all aspects of the work in ensuring that questions related to the accuracy or integrity of any part of the work are appropriately investigated and resolved.

Open Access Statement: This is an Open Access article distributed in accordance with the Creative Commons Attribution-NonCommercial-NoDerivs 4.0 International License (CC BY-NC-ND 4.0), which permits the noncommercial replication and distribution of the article with the strict proviso that no changes or edits are made and the original work is properly cited (including links to both the formal publication through the relevant DOI and the license). See: https://creativecommons.org/licenses/by-nc-nd/4.0/.

\section{References}

1. De A, Duseja A. Natural History of Simple Steatosis or Nonalcoholic Fatty Liver. J Clin Exp Hepatol 2020;10:255-62.

2. Bellentani S, Tiribelli C. Is it time to change NAFLD and NASH nomenclature? Lancet Gastroenterol Hepatol 2017;2:547-8.

3. Tilg H, Effenberger M. From NAFLD to MAFLD: when pathophysiology succeeds. Nat Rev Gastroenterol Hepatol 2020;17:387-8.

4. Younossi Z, Anstee QM, Marietti M, et al. Global burden of NAFLD and NASH: trends, predictions, risk factors and prevention. Nat Rev Gastroenterol Hepatol 2018;15:11-20.

5. Nobili V, Alisi A, Valenti L, et al. NAFLD in children: new genes, new diagnostic modalities and new drugs. Nat Rev Gastroenterol Hepatol 2019;16:517-30.

6. Rosso N, Chavez-Tapia NC, Tiribelli C, et al. Translational approaches: from fatty liver to non-alcoholic steatohepatitis. World J Gastroenterol 2014;20:9038-49.

7. Younossi ZM, Blissett D, Blissett R, et al. The economic and clinical burden of nonalcoholic fatty liver disease in the United States and Europe. Hepatology 2016;64:1577-86.

8. Angulo P, Kleiner DE, Dam-Larsen S, et al. Liver Fibrosis, but No Other Histologic Features, Is Associated With Long-term Outcomes of Patients With Nonalcoholic Fatty Liver Disease. Gastroenterology 2015;149:389-397.e10.

9. Kawada N, Imanaka K, Kawaguchi T, et al. Hepatocellular carcinoma arising from non-cirrhotic nonalcoholic steatohepatitis. J Gastroenterol 2009;44:1190-4.

10. Paradis V, Zalinski S, Chelbi E, et al. Hepatocellular carcinomas in patients with metabolic syndrome often develop without significant liver fibrosis: a pathological analysis. Hepatology 2009;49:851-9.

11. Chalasani N, Younossi Z, Lavine JE, et al. The diagnosis and management of non-alcoholic fatty liver disease: Practice Guideline by the American Association for the Study of Liver Diseases, American College of Gastroenterology, and the American Gastroenterological Association. Hepatology 2012;55:2005-23.

12. Puri P, Baillie RA, Wiest MM, et al. A lipidomic analysis of nonalcoholic fatty liver disease. Hepatology 2007;46:1081-90.

13. Anjani K, Lhomme M, Sokolovska N, et al. Circulating phospholipid profiling identifies portal contribution to NASH signature in obesity. J Hepatol 2015;62:905-12.

14. Han MAT, Saouaf R, Ayoub W, et al. Magnetic resonance imaging and transient elastography in the management of Nonalcoholic Fatty Liver Disease (NAFLD). Expert Rev Clin Pharmacol 2017;10:379-90.

15. Kleiner DE, Brunt EM, Van Natta M, et al. Design and validation of a histological scoring system for nonalcoholic fatty liver disease. Hepatology 2005;41:1313-21. 
16. Younossi ZM, Stepanova M, Rafiq N, et al. Pathologic criteria for nonalcoholic steatohepatitis: interprotocol agreement and ability to predict liver-related mortality. Hepatology 2011;53:1874-82.

17. Ekstedt M, Franzén LE, Mathiesen UL, et al. Low clinical relevance of the nonalcoholic fatty liver disease activity score (NAS) in predicting fibrosis progression. Scand J Gastroenterol 2012;47:108-15.

18. Bedossa P, Poitou C, Veyrie N, et al. Histopathological algorithm and scoring system for evaluation of liver lesions in morbidly obese patients. Hepatology 2012;56:1751-9.

19. Ekstedt M, Hagström H, Nasr P, et al. Fibrosis stage is the strongest predictor for disease-specific mortality in NAFLD after up to 33 years of follow-up. Hepatology 2015;61:1547-54.

20. Bedogni G, Bellentani S, Miglioli L, et al. The Fatty Liver Index: a simple and accurate predictor of hepatic steatosis in the general population. BMC Gastroenterol 2006;6:33.

21. Poynard T, Ratziu V, Naveau S, et al. The diagnostic value of biomarkers (SteatoTest) for the prediction of liver steatosis. Comp Hepatol 2005;4:10.

22. Kotronen A, Peltonen M, Hakkarainen A, et al. Prediction of non-alcoholic fatty liver disease and liver fat using metabolic and genetic factors. Gastroenterology 2009;137:865-72.

23. Angulo P, Hui JM, Marchesini G, et al. The NAFLD fibrosis score: a noninvasive system that identifies liver fibrosis in patients with NAFLD. Hepatology 2007;45:846-54.

24. Sumida Y, Yoneda M, Hyogo H, et al. Validation of the FIB4 index in a Japanese nonalcoholic fatty liver disease population. BMC Gastroenterol 2012;12:2.

25. Guha IN, Parkes J, Roderick P, et al. Noninvasive markers of fibrosis in nonalcoholic fatty liver disease: Validating the European Liver Fibrosis Panel and exploring simple markers. Hepatology 2008;47:455-60.

26. Lassailly G, Caiazzo R, Hollebecque A, et al. Validation of noninvasive biomarkers (FibroTest, SteatoTest, and NashTest) for prediction of liver injury in patients with morbid obesity. Eur J Gastroenterol Hepatol 2011;23:499-506.

27. Darmawan G, Hamijoyo L, Hasan I. Association between Serum Uric Acid and Non-Alcoholic Fatty Liver Disease: A Meta-Analysis. Acta Med Indones 2017;49:136-47.

28. Liu Z, Que S, Zhou L, et al. Dose-response Relationship of Serum Uric Acid with Metabolic Syndrome and Nonalcoholic Fatty Liver Disease Incidence: A Meta-analysis of Prospective Studies. Sci Rep 2015;5:14325.
29. Wu SJ, Zhu GQ, Ye BZ, et al. Association between sexspecific serum uric acid and non-alcoholic fatty liver disease in Chinese adults: a large population-based study. Medicine (Baltimore) 2015;94:e802.

30. Yang H, Li D, Song X, et al. Joint associations of serum uric acid and ALT with NAFLD in elderly men and women: a Chinese cross-sectional study. J Transl Med 2018;16:285.

31. Gurian E, Giraudi P, Rosso N, et al. Differentiation between stages of non-alcoholic fatty liver diseases using surface-enhanced Raman spectroscopy. Anal Chim Acta [Internet]. 2020 Feb 22 [cited 2020 Feb 28]; Available online: http://www.sciencedirect.com/science/article/pii/ S0003267020302300

32. Feldstein AE, Wieckowska A, Lopez AR, et al. Cytokeratin-18 fragment levels as noninvasive biomarkers for nonalcoholic steatohepatitis: a multicenter validation study. Hepatology 2009;50:1072-8.

33. Musso G, Gambino R, Cassader M, et al. Meta-analysis: natural history of non-alcoholic fatty liver disease (NAFLD) and diagnostic accuracy of non-invasive tests for liver disease severity. Ann Med 2011;43:617-49.

34. Shen J, Chan HLY, Wong GLH, et al. Non-invasive diagnosis of non-alcoholic steatohepatitis by combined serum biomarkers. J Hepatol 2012;56:1363-70.

35. Machado MV, Coutinho J, Carepa F, et al. How adiponectin, leptin, and ghrelin orchestrate together and correlate with the severity of nonalcoholic fatty liver disease. Eur J Gastroenterol Hepatol 2012;24:1166-72.

36. Younossi ZM, Jarrar M, Nugent C, et al. A novel diagnostic biomarker panel for obesity-related nonalcoholic steatohepatitis (NASH). Obes Surg 2008;18:1430-7.

37. Tamimi TIAR, Elgouhari HM, Alkhouri N, et al. An apoptosis panel for nonalcoholic steatohepatitis diagnosis. J Hepatol 2011;54:1224-9.

38. Trak-Smayra V, Dargere D, Noun R, et al. Serum proteomic profiling of obese patients: correlation with liver pathology and evolution after bariatric surgery. Gut 2009;58:825-32.

39. Miller MH, Walsh SV, Atrih A, et al. Serum proteome of nonalcoholic fatty liver disease: a multimodal approach to discovery of biomarkers of nonalcoholic steatohepatitis. J Gastroenterol Hepatol 2014;29:1839-47.

40. Tanwar S, Trembling PM, Guha IN, et al. Validation of terminal peptide of procollagen III for the detection and assessment of nonalcoholic steatohepatitis in patients with nonalcoholic fatty liver disease. Hepatology 2013;57:103-11. 
41. Boyle M, Tiniakos D, Schattenberg JM, et al. Performance of the PRO-C3 collagen neo-epitope biomarker in nonalcoholic fatty liver disease. JHEP Rep 2019;1:188-98.

42. Liu CH, Ampuero J, Gil-Gómez A, et al. miRNAs in patients with non-alcoholic fatty liver disease: A systematic review and meta-analysis. J Hepatol 2018;69:1335-48.

43. López-Riera M, Conde I, Quintas G, et al. Noninvasive prediction of NAFLD severity: a comprehensive, independent validation of previously postulated serum microRNA biomarkers. Sci Rep 2018;8:10606.

44. Hegazy M, Saleh SA, Ezzat A, et al. Novel Application of the Traditional Lipid Ratios as Strong Risk Predictors of NASH. Diabetes Metab Syndr Obes 2020;13:297-305.

45. He L, Deng L, Zhang Q, et al. Diagnostic Value of CK18, FGF-21, and Related Biomarker Panel in Nonalcoholic Fatty Liver Disease: A Systematic Review and MetaAnalysis. BioMed Res Int 2017;2017:9729107.

46. Lădaru A, Bălănescu P, Stan M, et al. Candidate proteomic biomarkers for non-alcoholic fatty liver disease (steatosis and non-alcoholic steatohepatitis) discovered with mass-spectrometry: a systematic review. Biomarkers 2016;21:102-14

47. Rodríguez-Suárez E, Duce AM, Caballería J, et al. Nonalcoholic fatty liver disease proteomics. Proteomics Clin Appl 2010;4:362-71.

48. Lin B, Ma Y, Wu S, et al. Novel Serum Biomarkers for Noninvasive Diagnosis and Screening of Nonalcoholic Fatty Liver Disease-Related Hepatic Fibrosis. OMICS 2019;23:181-9.

49. Hou W, Janech MG, Sobolesky PM, et al. Proteomic screening of plasma identifies potential noninvasive biomarkers associated with significant/advanced fibrosis in patients with nonalcoholic fatty liver disease. Biosci Rep 2020;40:BSR20190395.

50. Lai M, Libermann T, Charlton M, et al. Evaluation of SOMAscan as a discovery platform to identify noninvasive protein biomarkers for diagnosis and monitoring of NASH. J Hepatol 2018;68:S556.</jrn>

51. Cai C, Lin Y, Yu C. Circulating miRNAs as Novel Diagnostic Biomarkers in Nonalcoholic Fatty Liver Disease: A Systematic Review and Meta-Analysis. Can J Gastroenterol Hepatol 2019;2019:2096161.

52. Wong VWS. Predicting NASH response with liver fat: Are we back to square one? J Hepatol 2020;72:386-8.

53. Hodson L, Parry SA, Cornfield T, et al. Using total plasma triacylglycerol to assess hepatic de novo lipogenesis as an alternative to VLDL triacylglycerol. Ups J Med Sci 2020;125:211-6.
54. Mayo R, Crespo J, Martínez-Arranz I, et al. Metabolomicbased noninvasive serum test to diagnose nonalcoholic steatohepatitis: Results from discovery and validation cohorts. Hepatol Commun 2018;2:807-20.

55. Chiappini F, Coilly A, Kadar H, et al. Metabolism dysregulation induces a specific lipid signature of nonalcoholic steatohepatitis in patients. Sci Rep 2017;7:46658.

56. Garcia-Jaramillo M, Spooner MH, Löhr CV, et al. Lipidomic and transcriptomic analysis of western dietinduced nonalcoholic steatohepatitis (NASH) in female Ldlr -/- mice. PLoS One 2019;14:e0214387.

57. Jha P, McDevitt MT, Halilbasic E, et al. Genetic Regulation of Plasma Lipid Species and Their Association with Metabolic Phenotypes. Cell Syst 2018;6:709-721.e6.

58. Gorden DL, Myers DS, Ivanova PT, et al. Biomarkers of NAFLD progression: a lipidomics approach to an epidemic. J Lipid Res 2015;56:722-36.

59. Perakakis N, Polyzos SA, Yazdani A, et al. Non-invasive diagnosis of non-alcoholic steatohepatitis and fibrosis with the use of omics and supervised learning: A proof of concept study. Metabolism 2019;101:154005.

60. Poupin N, Vinson F, Moreau A, et al. Improving lipid mapping in Genome Scale Metabolic Networks using ontologies. Metabolomics 2020;16:44.

61. Ščupáková K, Soons Z, Ertaylan G, et al. Spatial Systems Lipidomics Reveals Nonalcoholic Fatty Liver Disease Heterogeneity. Anal Chem 2018;90:5130-8.

62. Puri P, Wiest MM, Cheung O, et al. The plasma lipidomic signature of nonalcoholic steatohepatitis. Hepatology 2009;50:1827-38.

63. Loomba R, Quehenberger O, Armando A, et al. Polyunsaturated fatty acid metabolites as novel lipidomic biomarkers for noninvasive diagnosis of nonalcoholic steatohepatitis. J Lipid Res 2015;56:185-92.

64. Santoleri D, Titchenell PM. Resolving the Paradox of Hepatic Insulin Resistance. Cell Mol Gastroenterol Hepatol 2019;7:447-56.

65. Verhelst X, Dias AM, Colombel JF, et al. Protein Glycosylation as a Diagnostic and Prognostic Marker of Chronic Inflammatory Gastrointestinal and Liver Diseases. Gastroenterology 2020;158:95-110.

66. Kamada Y, Akita M, Takeda Y, et al. Serum Fucosylated Haptoglobin as a Novel Diagnostic Biomarker for Predicting Hepatocyte Ballooning and Nonalcoholic Steatohepatitis. PLoS One 2013;8:e66328.

67. Blomme B, Francque S, Trépo E, et al. N-glycan based biomarker distinguishing non-alcoholic steatohepatitis 
from steatosis independently of fibrosis. Dig Liver Dis 2012;44:315-22.

68. Vanderschaeghe D, Laroy W, Sablon E, et al. GlycoFibroTest is a highly performant liver fibrosis biomarker derived from DNA sequencer-based serum protein glycomics. Mol Cell Proteomics 2009;8:986-94.

69. Callewaert N, Van Vlierberghe H, Van Hecke A, et al. Noninvasive diagnosis of liver cirrhosis using DNA sequencer-based total serum protein glycomics. Nat Med 2004;10:429-34.

70. Debruyne EN, Vanderschaeghe D, Van Vlierberghe $\mathrm{H}$, et al. Diagnostic value of the hemopexin N-glycan profile in hepatocellular carcinoma patients. Clin Chem 2010;56:823-31.

71. Liu XE, Desmyter L, Gao CF, et al. N-glycomic changes in hepatocellular carcinoma patients with liver cirrhosis induced by hepatitis B virus. Hepatology 2007;46:1426-35.

72. Verhelst X, Geerts A, Jochmans I, et al. Glycome Patterns of Perfusate in Livers Before Transplantation Associate With Primary Nonfunction. Gastroenterology 2018;154:1361-8.

73. Ogawa K, Kobayashi T, Furukawa JI, et al. Tri-antennary tri-sialylated mono-fucosylated glycan of alpha-1

Cite this article as: Rosso N, Stephenson AM, Giraudi PJ, Tiribelli C. Diagnostic management of nonalcoholic fatty liver disease: a transformational period in the development of diagnostic and predictive tools-a narrative review. Ann Transl Med 2021;9(8):727. doi: 10.21037/atm-20-4723 antitrypsin as a non-invasive biomarker for non-alcoholic steatohepatitis: a novel glycobiomarker for non-alcoholic steatohepatitis. Sci Rep 2020;10:321.

74. Blomme B, Fitzpatrick E, Quaglia A, et al. Serum protein $\mathrm{N}$-glycosylation in paediatric non-alcoholic fatty liver disease. Pediatr Obes 2012;7:165-73.

75. Dyson JK, Anstee QM, McPherson S. Non-alcoholic fatty liver disease: a practical approach to diagnosis and staging. Frontline Gastroenterol 2014;5:211-8.

76. Chen C, Schmilovitz-Weiss H, Liu X, et al. Serum protein $\mathrm{N}$-glycans profiling for the discovery of potential biomarkers for nonalcoholic steatohepatitis. J Proteome Res 2009;8:463-70.

77. Blomme B, Van Steenkiste C, Callewaert N, et al. Alteration of protein glycosylation in liver diseases. J Hepatol 2009;50:592-603.

78. Gorowska-Kowolik K, Chobot A, Kwiecien J. 13C Methacetin Breath Test for Assessment of Microsomal Liver Function: Methodology and Clinical Application. Gastroenterol Res Pract 2017;2017:7397840.

79. Saponaro C, Gaggini M, Carli F, et al. The Subtle Balance between Lipolysis and Lipogenesis: A Critical Point in Metabolic Homeostasis. Nutrients 2015;7:9453-74. 